\title{
Semi analytical method for calculating Dean's vortex in Torus of elliptical cross section
}

\author{
S. Ajgoun ${ }^{1}$, J. Khalid Naciri, R. Khatyr \\ Laboratory of Mechanics, Faculty of Sciences Aïn Chock, B.P. 5366, Maarif, Hassan II University, Casablanca 20100, \\ Morocco \\ Corresponding authors : E-mail : $\left({ }^{1}\right)$ s.ajgoun@outlook.fr
}

\begin{abstract}
Based on the work of Dean (1927 and 1928) [1-2] and Cuming (1952) [3], the stationary flow of an incompressible Newtonian fluid through a curved pipe of uniform curvature and with elliptic cross section is studied. The Navier-Stokes equations are expressed in toroïdal coordinates system $(s, r, \theta)$. Following Dean's approach, the governing equations for the fluid motion through a curved elliptical channel are solved by using an original semi analytical method for the resolution of a biharmonic equation. The main interest in this study is to test and validate in the case of an elliptical cross section the proposed semi-analytical method. The latter can then be used for other geometries for which explicit solutions are not available.
\end{abstract}

Keywords : Toroïdal coordinates, Curvature, Curved pipes, Centrifugal force, Dean vortex.

\section{Introduction}

Recently, in the domain of microfluidics [4], a particular interest is brought to the flows in curved pipes of various cross sectional shapes because of their technical importance, for example in fluid transport industry, and heat transfer : helical and spiral coils are often used for transferring heat in mixing and storage, as well as for heat exchangers.

Many studies have focused on the study of flows in complex geometries in different situations. Therefore, Dean [1] is the first who gave a mathematical proof of the existence of secondary flow, for flow through a curved pipe with constant curvature. The technique used was to assume that the secondary flow is just a small disturbance of the Poiseuille flow in a straight tube. He analyzed theoretically for low curvature pipes and fully developed laminar flow, the dependence of the movement of the fluid particles to a dimensionless dynamic parameter characterizing the secondary motion noted $D_{n}=$ $\left(D_{h} / 2 R_{c}\right)^{1 / 2} R e$, $\left(D_{n}\right.$ : Dean number, $D_{h}$ : hydraulic diameter, $R_{c}$ : radius of curvature, Re : Reynolds number). In 1928 [2], Dean showed in his second article that the pressure gradient and the flow rate through a curved tube depends on the curvature. A theoretical analysis was made by Cuming [3] in 1952, where he studied analytically the flow of an incompressible viscous fluid through curved pipes of different sections (circular, elliptical, square). Wang in 1980 [5] gave an exact solution for the flow through narrow with constant curvature and he used a perturbation scheme for the case of small, periodic curvature. Germano in 1982 [6] analyzed the effect of torsion on a helical pipe flow.

An analytical and numerical analysis of the effect of the variation in curvature and torsion on the steady and unsteady flows in weakly curved pipes of different four pipe shapes was treated by Gammack [7] in 2001. Siggers in 2005 [8] focused in his research on blood circulation in a curved artery and studied the effects of centrifugal forces and Coriolis forces on flows in pipes of finite curvature.

The aim of this study, based on Dean's approach, is to use an original semi analytical method based on General Michell solution for the biharmonic equation in polar coordinates [9], for solving the governing equations of the 3D-flow of an incompressible fluid in a pipe for uniform curvature and with elliptical section.

\section{Formulation of problem}

Using the orthogonal coordinate system $R(0, \vec{x}, \vec{y}, \vec{z})$, we consider the steady flow of an incompressible Newtonian fluid in a curved pipe of elliptical cross section with uniform curvature. We define the orthonormal triad $(\vec{\tau}, \vec{N}, \vec{B})$ where $\vec{\tau}$ is the tangent vector, $\vec{N}$ the normal vector and $\vec{B}$ the binormal vector, as shown in Fig.1. Let the velocity components along these axes be $u, v$, and $w$.

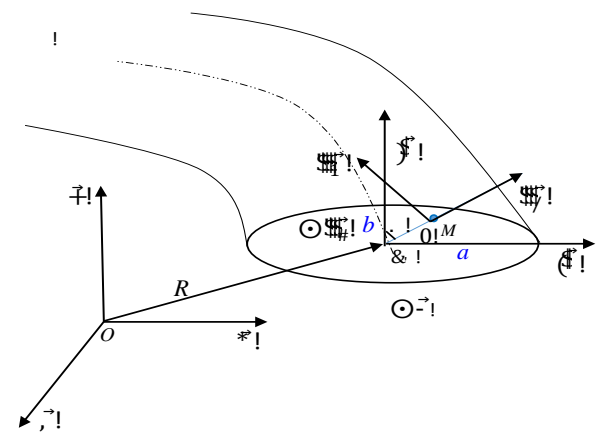

Fig. 1 The coordinate system

As shown in figure $1, a$ and $b$ are respectively the major axis and minor axis of the elliptic cross section, $\theta$ is the angle between the normal vector and the vector $\overrightarrow{\mathrm{O}_{1} M}$, 
$R_{F}\left(O_{1}, \vec{\tau}, \vec{N}, \vec{B}\right)$ is the coordinate system located at the center of the cross-section and $\overrightarrow{\mathrm{R}}=\overrightarrow{\mathrm{OO}_{1}}$ is position vector of the center $\mathrm{O}_{1}$ in $R(O, \vec{x}, \vec{y}, \vec{z})$. Position of any point in the cross section of the tube is given by the polar coordinates $(r, \theta)$ in the plane $(\vec{N}, \vec{B})$ (with : $r=\|\overrightarrow{O M}\|$ ). By using the non-dimensionalized variables :

$$
\begin{gathered}
u=U u^{*}, v=V v^{*}, w=V w^{*}, p=p_{0} p^{*}, s=R_{m} s^{*}, \\
r=a r^{*}
\end{gathered}
$$

Where $U$ and $V$ are reference velocity, $p_{0}$ is a reference pressure, $R_{m}$ is the characteristic length, and $a$ is the radius of curvature.

and by considering the case where $\varepsilon=a / R_{c} \ll 1$, the governing equations for the steady flow of a viscous incompressible fluid may be restated in the following dimensionless forms :

Continuity Equation :

$$
\frac{\partial\left(r^{*} v_{1}^{*}\right)}{\partial r^{*}}+\frac{\partial w_{1}^{*}}{\partial \theta}=0
$$

Momentum equation for secondary flow

$\overrightarrow{\boldsymbol{e}_{s}}$ :

$D_{n}\left(v^{*} \frac{\partial u^{*}}{\partial r^{*}}+\frac{w^{*}}{r^{*}} \frac{\partial u^{*}}{\partial \theta}\right)=-\left\{\frac{p_{0} a}{v U R_{m}}\right\} \frac{\partial p^{*}}{\partial s^{*}}+\frac{1}{r^{*}} \frac{\partial}{\partial r^{*}}\left(r^{*} \frac{\partial u^{*}}{\partial r^{*}}\right)+$

$\frac{1}{r^{*}} \frac{\partial^{2} u^{*}}{\partial \theta^{2}}$

$\overrightarrow{\boldsymbol{e}_{r}}$ :

$$
\begin{aligned}
& D_{n}\left(v^{*} \frac{\partial v^{*}}{\partial r^{*}}+\frac{w^{*}}{r^{*}} \frac{\partial v^{*}}{\partial \theta}-\frac{w^{* 2}}{r^{*}}+\frac{u^{* 2} \cos \theta}{R_{c}^{*}}\right)=-\left\{\frac{p_{0} a}{v V}\right\} \frac{\partial p^{*}}{\partial r^{*}}- \\
& \frac{1}{r^{*}} \frac{\partial}{\partial \theta}\left(\frac{\partial w^{*}}{\partial r^{*}}+\frac{w^{*}}{r^{*}}-\frac{1}{r^{*}} \frac{\partial v^{*}}{\partial \theta}\right)
\end{aligned}
$$

$\overrightarrow{\boldsymbol{e}_{\theta}}$ :

$$
\begin{aligned}
& D_{n}\left(v^{*} \frac{\partial w^{*}}{\partial r^{*}}+\frac{w^{*}}{r^{*}} \frac{\partial w^{*}}{\partial \theta}+\frac{v^{*} w^{*}}{r^{*}}-\frac{u^{* 2} \sin \theta}{R_{c}^{*}}\right)= \\
& -\left\{\frac{p_{0} a}{v V}\right\} \frac{1}{r^{*}} \frac{\partial p^{*}}{\partial \theta}+\frac{\partial}{\partial r^{*}}\left(\frac{\partial w^{*}}{\partial r^{*}}+\frac{w^{*}}{r^{*}}-\frac{1}{r^{*}} \frac{\partial v^{*}}{\partial \theta}\right)
\end{aligned}
$$

The non slip Boundary conditions are $v=w=0$ at the wall.

Where $\mathrm{D}_{\mathrm{n}}$ is the Dean number defined by:

\section{Resolution}

$$
D_{n}=\operatorname{Re} \sqrt{\varepsilon}=\frac{U a}{v} \sqrt{\varepsilon}
$$

Following the approach of Dean, the solutions of equations (1-4) with their associated boundary conditions is sought in the form of asymptotic expansions in $D_{n}$ :

$$
\begin{aligned}
& u^{*}=u_{0}{ }^{*}\left(r^{*}, \theta\right)+D_{n} u_{1}^{*}\left(r^{*}, \theta\right)+D_{n}{ }^{2} u_{2}^{*}\left(r^{*}, \theta\right)+. . \\
& v^{*}=0+D_{n} v_{1}^{*}\left(r^{*}, \theta\right)+D_{n}{ }^{2} v_{2}{ }^{*}\left(r^{*}, \theta\right)+. . \\
& w^{*}=0+D_{n} w_{1}^{*}\left(r^{*}, \theta\right)+D_{n}{ }^{2} w_{2}{ }^{*}\left(r^{*}, \theta\right)+. . \\
& p^{*}=p_{0}{ }^{*}+D_{n} p_{1}^{*}\left(r^{*}, \theta\right)+D_{n}{ }^{2} p_{2}^{*}\left(r^{*}, \theta\right)+. .
\end{aligned}
$$

At zero order in $D_{n}$, we have :

$$
\left\{\begin{array}{l}
0=-\frac{\partial p_{0}^{*}}{\partial s^{*}}+\frac{1}{r^{*}} \frac{\partial}{\partial r^{*}}\left[r^{*} \frac{\partial}{\partial r^{*}}\left(u_{0}^{*}\right)\right]+\frac{1}{r^{* 2}} \frac{\partial^{2}}{\partial \theta^{2}}\left(u_{0}^{*}\right) \\
0=-\frac{\partial p_{0}^{*}}{\partial r^{*}} \\
0=-\frac{1}{r^{*}} \frac{\partial p_{0}^{*}}{\partial \theta}
\end{array}\right.
$$

The profile velocity satisfying (6) and the no slip boundary conditions can be written as [10] :

$$
u_{0}^{*}\left(r^{*}, \theta\right)=\frac{2 Q^{*}}{\pi a b}\left(1-\frac{r^{* 2} \sin ^{2} \theta}{a^{2}}-\frac{r^{* 2} \cos ^{2} \theta}{b^{2}}\right)
$$

Where $Q^{*}$ is the dimensionless flow rate.

At first order in $D_{n}$, the system (1-4) reduces to :

$$
\begin{gathered}
\frac{\partial\left(r^{*} v_{1}^{*}\right)}{\partial r^{*}}+\frac{\partial w_{1}^{*}}{\partial \theta}=0 \\
0=-\frac{\partial p_{1}^{*}}{\partial s^{*}}+\frac{\partial^{2} u_{1}^{*}}{\partial r^{* 2}}+\frac{1}{r^{*}} \frac{\partial u_{1}^{*}}{\partial r^{*}}+\frac{1}{r^{*}} \frac{\partial^{2} u_{1}^{*}}{\partial \theta^{2}} \\
\frac{u_{0}^{* 2} \sin \theta}{R_{c}}=-\left\{\frac{p_{0} a}{v V}\right\} \frac{\partial p_{1}^{*}}{\partial r^{*}}-\frac{1}{r^{*}} \frac{\partial}{\partial \theta}\left(\frac{\partial w_{1}^{*}}{\partial r^{*}}+\frac{w_{1}^{*}}{r^{*}}-\frac{1}{r^{*}} \frac{\partial v_{1}^{*}}{\partial \theta}\right) \\
\frac{u_{0}^{* 2} \cos \theta}{R_{c}}=-\left\{\frac{p_{0} a}{v V}\right\} \frac{1}{r^{*}} \frac{\partial p_{1}^{*}}{\partial \theta}+\frac{\partial}{\partial r^{*}}\left(\frac{\partial w_{1}^{*}}{\partial r^{*}}+\frac{w_{1}^{*}}{r^{*}}-\frac{1}{r^{*}} \frac{\partial v_{1}^{*}}{\partial \theta}\right)
\end{gathered}
$$

Equation (8) implies the existence of a dimensionless pseudo-stream function defined in such a way that :

$$
v_{1}^{*}=\frac{1}{r^{*}} \frac{\partial \psi_{1}^{*}}{\partial \theta}, w_{1}^{*}=-\frac{\partial \psi_{1}^{*}}{\partial r^{*}}
$$

Inserting these expressions and eliminating the pressure terms between equations (10) and (11), give the following equation for $\psi_{1}^{*}$ :

$$
\begin{gathered}
\Delta\left(\Delta \psi_{1}^{*}\right)=\frac{16 Q^{* 2}}{b^{2}(\pi a b)^{2} R_{c}}[ \\
{\left[\frac{1}{*} \cos \theta-\left(\frac{1}{4 a^{2}}+\frac{3}{4 b^{2}}\right) r^{* 3} \cos \theta+\right.} \\
\left.\left(\frac{1}{4 a^{2}}-\frac{1}{4 b^{2}}\right) r^{* 3} \cos 3 \theta\right]
\end{gathered}
$$

With the boundary conditions $\frac{\partial \psi_{1}^{*}}{\partial r^{*}}=\frac{\partial \psi_{1}^{*}}{\partial \theta}=0$ at the wall. After calculating the particular solutions and taking into account the Michel's [9] solution for the bihamonic equations in polar coordinates to establish the solution of the homogeneous equation, we obtain the following form for the solution of (12) :

$\psi_{1}^{*}\left(r^{*}, \theta\right)=\frac{16 Q^{* 2}}{b^{2}(\pi a b)^{2} R_{c}}\left(\frac{r^{* 5} \cos \theta}{192}-\left(\frac{1}{4 a^{2}}+\frac{3}{4 b^{2}}\right) \frac{r^{* 7} \cos \theta}{1152}+\right.$ $\left(\frac{1}{4 a^{2}}-\frac{1}{4 b^{2}}\right) \frac{r^{* 7} \cos 3 \theta}{640}+\sum_{n=1}^{p}\left[\left(A_{n} r^{* n}+\right.\right.$ $\left.\left.\left.B_{n} r^{* n+2}\right) \cos (n \theta)+\left(C_{n} r^{* n}+D_{n} r^{* n+2}\right) \sin (n \theta)\right]\right)$

$A_{n}, B_{n}, C_{n}, D_{n}$ are real constants to be determined.

The main difficulty in solving a biharmonic equation lies in obtaining integration constants that allow the solution to satisfy zero velocity conditions at the wall. For this purpose, the proposed method consists of decomposing the whole expression (13) at the wall in Fourier series, then by canceling the contribution of each of the harmonics at the wall, we obtain a system of algebraic equations whose resolution allows to fix the values of integration constants to a given order in $\mathrm{p}$. In the case of an elliptic cross section of the torus, with semi axes a and b, we obtain:

$$
\begin{aligned}
& \psi_{1}^{*}\left(r^{*}, \theta\right)=\frac{16 Q^{* 2}}{b^{2}(\pi a b)^{2} R_{C}}\left(\frac{r^{* 5} \cos \theta}{192}-\left(\frac{1}{4 a^{2}}+\frac{3}{4 b^{2}}\right) \frac{r^{* 7} \cos \theta}{1152}+\right. \\
& \left(\frac{1}{4 a^{2}}-\frac{1}{4 b^{2}}\right) \frac{r^{* 7} \cos 3 \theta}{640}+\left(A_{1} r^{*}+\right. \\
& \left.B_{1} r^{* 3}\right) \cos \theta+\left(A_{3} r^{* 3}+B_{3} r^{* 5}\right) \cos 3 \theta+\left(A_{5} r^{* 3}+\right. \\
& \left.\left.B_{5} r^{* 7}\right) \cos 5 \theta+A_{7} r^{* 7} \cos 7 \theta\right)
\end{aligned}
$$


Where :

$A_{1}=\frac{375 a^{12} b^{4}+820 a^{10} b^{6}+1114 a^{8} b^{8}+212 a^{6} b^{10}+39 a^{4} b^{12}}{360\left(5 a^{4}+2 a^{2} b^{2}+b^{4}\right) G}$

$B_{1}$

$=-\frac{275 a^{12} b^{2}+725 a^{10} b^{4}+1030 a^{8} b^{6}+434 a^{6} b^{8}+87 a^{4} b^{10}+9 b^{12}}{160\left(5 a^{4}+2 a^{2} b^{2}+b^{4}\right) G}$

$A_{3}$

$=-\frac{\left(a^{2}-b^{2}\right)\left(275 a^{10} b^{2}+500 a^{8} b^{4}+730 a^{6} b^{6}+132 a^{4} b^{8}+27 a^{2} b^{10}\right)}{480\left(5 a^{4}+2 a^{2} b^{2}+b^{4}\right) G}$

$B_{3}$

$=\frac{\left(a^{2}-b^{2}\right)\left(5 a^{2}+3 b^{2}\right)\left(175 a^{8}+340 a^{6} b^{2}+410 a^{4} b^{4}+84 a^{2} b^{6}+15 b^{8}\right)}{1920\left(5 a^{4}+2 a^{2} b^{2}+b^{4}\right) G}$

$A_{5}$

$$
\begin{gathered}
=\frac{(a-b)^{2}(a+b)^{2}\left(175 a^{8}+180 a^{6} b^{2}+346 a^{4} b^{4}+52 a^{2} b^{6}+15 b^{8}\right)}{1920\left(5 a^{4}+2 a^{2} b^{2}+b^{4}\right) G} \\
B_{5}=-\frac{(a-b)^{2}(a+b)^{2}\left(105 a^{6}+77 a^{4} b^{2}-5 a^{2} b^{4}+15 b^{6}\right)}{23040\left(5 a^{4}+2 a^{2} b^{2}+b^{4}\right) G} \\
A_{7}=-\frac{(a-b)^{3}(a+b)^{3}\left(15 a^{4}-34 a^{2} b^{2}+3 b^{4}\right)}{23040 a^{2} b^{2} G} \\
G=\left(35 a^{8}+84 a^{6} b^{2}+114 a^{4} b^{4}+20 a^{2} b^{6}+3 b^{8}\right)
\end{gathered}
$$

From this latter expression, we directly obtain analytically the expressions of the velocities of the secondary flow using the relations $v_{1}^{*}=\frac{1}{r^{*}} \frac{\partial \psi_{1}^{*}}{\partial \theta}, w_{1}^{*}=-\frac{\partial \psi_{1}^{*}}{\partial r^{*}}$.

We also note that in the case of a circular section $(\mathrm{a}=\mathrm{b}=$ $1)$, the relation (14) is identical to the expression established by Dean given by :

$\psi_{1}^{*}\left(r^{*}, \theta\right)=\frac{16 Q^{* 2}}{(\pi)^{2} R_{C}}\left[\frac{r^{*}}{288}-\frac{r^{* 3}}{128}+\frac{r^{* 5}}{192}-\frac{r^{* 7}}{1152}\right] \cos \theta$

\section{Results}

Fig. 2 show the axial, radial and transversal velocity in the case for uniform curvature with $a=2, b=1$ and $Q^{*}=1$, the results shows that there is a secondary flow for curved elliptic channel as shown by the plot of the streamlines for the flow, and that the obtained results are in total agreement with those of Cumming [3] .

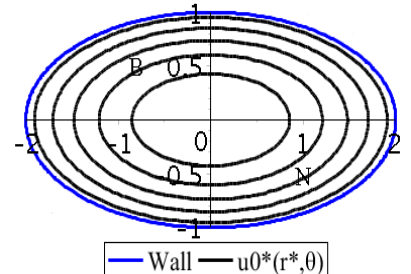

(a)

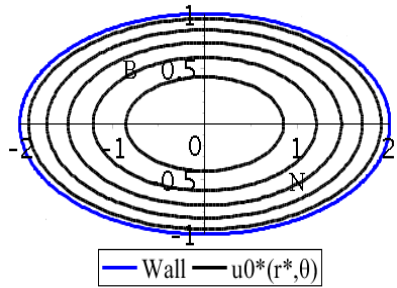

(c)

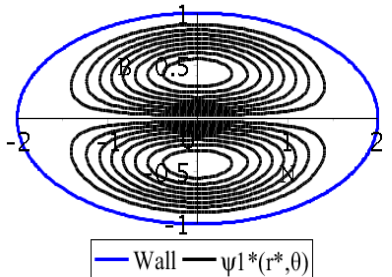

(b)

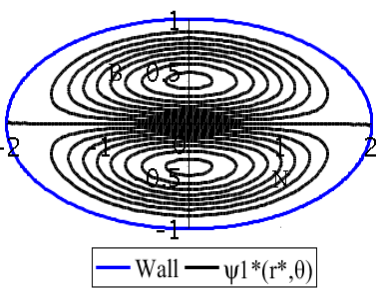

(d)
Fig. 2 : Contour plots of components of $u_{0}^{*}$ and $\psi_{1}^{*}:$ (a) Velocity axial at $\mathrm{O}\left(\mathrm{D}_{\mathrm{n}}^{0}\right)$ with $\mathrm{a}=2 \mathrm{~b}$; (b) Solution at $\mathrm{O}\left(\mathrm{D}_{\mathrm{n}}^{1}\right)$ with $\mathrm{a}=2 \mathrm{~b}$ (Semi-analytical method); (c) Velocity axial at $\mathrm{O}\left(\mathrm{D}_{\mathrm{n}}^{0}\right)$ with $\mathrm{a}=2 \mathrm{~b}$; (d) Solution at $\mathrm{O}\left(\mathrm{D}_{\mathrm{n}}^{1}\right)$ with $\mathrm{a}=2 \mathrm{~b}$ (Cuming)

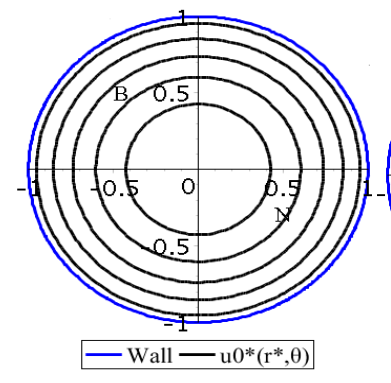

(a)

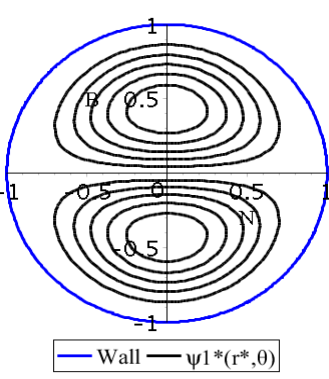

(b)
Fig. 3 : Contour plots of components of $u_{0}^{*}$ and $\psi_{1}^{*}$ with $\mathrm{a}=\mathrm{b}=1$ : (a) Velocity axial at $\mathrm{O}\left(\mathrm{D}_{\mathrm{n}}^{0}\right)$ (Poiseuille flow), (b) Solution at $\mathrm{O}\left(\mathrm{D}_{\mathrm{n}}^{1}\right)$ (Dean flow)

\section{Conclusion}

The main interest of the proposed semi-analytical method, which give the exact result of Dean in the case of circular cross section and the same result as Cumming in the elliptic case is that the method is suitable for other geometries for which explicit solutions are not available.

\section{Acknowledgment}

This work is done with the financial support of the National Center for Scientific and Technical Research (CNRST).

\section{Références}

[1] W. R. Dean, Note on the notion of fluid in a curved pipe, Science-Phil. Mag. S7, 4(20) (1927) 77-85.

[2] W. R. Dean, The stream line Motion of Fluid in a curved Pipe, Phil. Mag. S7, 5(30) (1928) 673-695.

[3] H. G. Cuming, The Secondary Flow in Curved Pipes, Reports and Memoranda $\mathrm{N}^{\circ}$ 2880, February, 1952.

[4] R. Rasooli, O. K. Karaoğlu, B. Çetin, Simulation of Particle Separation Using Inirtial Microfluidics in a Spiral Microchannel for Biomedical Aplications, 5th Micro and Nano Flows Conference, 11-14 September Milan, Italy, 2016.

[5] C.-Y. Wang, Flow in Narrow Curved Channels, J. Applied Mech. 47 (1980) 7-10.

[6] M. Germano, On the effect of torsion on a helical pipe flow, J. Fluid Mech. 125 (1982) 1-8.

[7] D. Gammack and P.E. Hydon, Flow in pipes with non-uniform curvature and torsion, J. Fluid Mech. 433 (2001) 357-382.

[8] J.H. Siggers and S.L. Waters, Steady flows in pipes with finite curvature, Physics Fluids 17, 077102 (2005); 10.1063/1.1955547

[9] W. M. Lai, D. Rubin, and E. Krempl, Introduction to Continuum Mechanic, $3^{\text {rd }}$ ed., ButterworthHeinemann, 1999.

[10] J. Lekner, Viscous flow through pipes of various cross-sections, Eur. J. Phys. 28 (2007) 521-527. 